\title{
Jejum pré-operatório em pacientes hospitalizados
}

\author{
Preoperative fasting in hospitalized patients
}

Patrícia da Fonseca Flores ${ }^{1}$, Raquel Milani El Kik ${ }^{2}$

\begin{abstract}
RESUMO
Introdução: A resposta metabólica ao trauma cirúrgico é potencializada pelo jejum pré-operatório prolongado. Estudos evidenciam que a redução do tempo de jejum com a ingestão de líquidos com carboidratos, no pré-operatório, não resulta em aumento do risco de morbidade associada à anestesia.

Objetivo: Revisar sobre as atuais recomendações da literatura acerca do jejum pré-operatório em pacientes hospitalizados.

Materiais e Métodos: Realizou-se uma revisão de literatura a partir das bases de dados Medline/Pubmed, Lilacs e Embase, publicados no período de 2002 até 2012, com os termos de indexação: jejum, inanição, período pré-operatório e cuidado pré-operatório.

Resultados: Foram incluídos 20 estudos na revisão. Foram encontrados resultados referentes às consequências da abreviação do jejum pré-operatório, através do uso de uma solução de líquido enriquecida com carboidrato. Os resultados demonstraram que o uso da solução determina maior satisfação, menor irritabilidade, aumento do $\mathrm{pH}$ gástrico e principalmente, reduz a resposta catabólica ao estresse cirúrgico, com consequente melhora da recuperação pós-operatória.

Conclusão: Os resultados de trabalhos clínicos consistentes têm feito com que velhos paradigmas sejam questionados dando lugar a práticas mais modernas. Soluções que contenham carboidratos e outros nutrientes são promissoras quanto à melhora na resposta endócrino-metabólica ao trauma. A satisfação do paciente também deve ser considerada, e é maior quando se empregam períodos mais curtos de jejum no pré-operatório.
\end{abstract}

Palavras-chave: inanição; período pré-operatório; cuidados pré-operatórios.

\begin{abstract}
Introduction: The metabolic response to surgical trauma is potencialized by prolonged preoperative fasting. Studies show that reducing the duration of fasting with the intake of liquids containing carbohydrates in the preoperative period, does not result in increased risk of morbidity associated with anesthesia.

Objective: To review the current recommendations on the literature about preoperative fasting in hospitalized patients. Materials and Methods: We conducted a literature review study using the databases Medline/Pubmed, Embase and Lilacs, considering works published from 2002 until 2012, with the indexing terms: fasting, starvation, preoperative and preoperative care.

Results: Twenty studies were included in the review. Results were found regarding the consequences of reducing the preoperative fasting by using a solution of liquid enriched with carbohydrate. The results showed that the use of the solution determines greater satisfaction, less irritability, increased gastric $\mathrm{pH}$ and foremost reduces the catabolic response to surgical stress, with consequent improvement in postoperative recovery.

Conclusions: The results of consistent clinical studies have led to question old paradigms and to give way to more modern practices. Solutions containing carbohydrate and other nutrients are promising regarding the improvement in endocrine-metabolic response to trauma. Patient satisfaction should also be considered, and is larger when employing shorter periods of fasting preoperatively.
\end{abstract}

Keywords: starvation; preoperative period; preoperative care.

\footnotetext{
${ }^{1}$ Nutricionista. Especialista em Terapia Nutricional Parenteral e Enteral pela Faculdade de Enfermagem, Nutrição e Fisioterapia (FAENFI) da Pontifícia Universidade Católica do Rio Grande do Sul (PUCRS). Nutricionista assistencial do Hospital São Lucas da PUCRS.

${ }^{2}$ Nutricionista. Doutoranda em Gerontologia Biomédica/PUCRS. Professora Adjunta do Curso de Nutrição da FAENFI/PUCRS.
} 


\section{INTRODUÇÃO}

De acordo com a definição da língua portuguesa, o jejum pode ser caracterizado pela abstenção ou redução de alimentos em períodos ou dias determinados por preceito médico ${ }^{1}$. Já a inanição é um termo definido como a ausência total de ingestão ou administração de nutrientes, sendo mantida apenas a ingestão de água ${ }^{2}$.

No procedimento cirúrgico, o jejum noturno préoperatório foi instituído quando as técnicas anestésicas ainda eram rudimentares, a fim de prevenir complicações pulmonares associadas a vômitos $e$ aspirações do conteúdo gástrico com o objetivo de garantir o esvaziamento gástrico e evitar broncoaspiração no momento da indução anestésica (Síndrome de Mendelson) ${ }^{3}$.

A resposta metabólica ao trauma cirúrgico é potencializada pelo jejum pré-operatório prolongado. Após algumas horas de jejum, ocorre a diminuição dos níveis de insulina e, em consequência, aumento dos níveis de glucagon, determinando uma utilização rápida da pequena reserva de glicogênio que se encontra em maior parte no fígado. Em menos de 24 horas de jejum, o glicogênio hepático é totalmente consumido. Antes disso, porém, a gliconeogênese é ativada e a proteína muscular passa a ser utilizada provendo glicose para os tecidos que dependem exclusivamente dela como fonte de energia (sistema nervoso central, medula renal e eritrócitos) ${ }^{4}$. Além do aumento dos hormônios contrarreguladores, o jejum associado ao trauma desencadeia maior produção de mediadores inflamatórios que incrementam a resposta orgânica com inúmeros efeitos, tais como aumento da resistência insulínica, proteólise muscular, lipólise e, dependendo do porte da operação, gera resposta inflamatória sistêmica.

Estudo publicado, recentemente demonstrou que o jejum pré-operatório de oito horas é estendido com frequência. Pacientes permanecem em média 16 horas ou mais em jejum até o início do procedimento anestésico, por diversos motivos, como atraso no início das operações e adiamento para outro período do dia. Como consequência, a resposta orgânica, produzida pelo trauma, inicia-se muitas vezes com o jejum prolongado. $\mathrm{O}$ mesmo estudo, prospectivo $\mathrm{e}$ randomizado, mostrou que reduzir o tempo de jejum através da ingestão de líquidos com carboidratos (CHO), no pré-operatório, não resulta em aumento do risco de morbidade associada à anestesia. Várias Sociedades de Anestesia já modificaram suas diretrizes e passaram a recomendar essa rotina de abreviação do jejum que antecede operações eletivas. A Associação Americana de Anestesiologistas (ASA) recomenda guardar jejum para alimentos líquidos sem resíduos (clear liquids) de, pelo menos, duas horas antes das intervenções $^{5}$. As diretrizes da European Society of Parenteral and Enteral Nutrition (ESPEN) também recomendam a abreviação do jejum para duas horas, com uso de bebida contendo $\mathrm{CHO}^{6}$.

Sendo assim, o objetivo do estudo foi revisar as atuais recomendações da literatura acerca do jejum préoperatório em pacientes hospitalizados.

\section{MATERIAIS E MÉTODOS}

Foi realizada uma revisão de literatura orientada por uma questão norteadora: Quais as atuais recomendações da literatura acerca do jejum préoperatório em pacientes hospitalizados? A revisão foi realizada por meio de buscas nas bases de dados Medline/Pubmed, Lilacs e Embase nos idiomas português, espanhol e inglês, publicados no período de 2002 até 2012. Incluíram-se guidelines, ensaios clínicos randomizados, controlados e meta-análises com as palavras-chave indexadas pela Bireme: jejum, inanição, período pré-operatório, cuidado pré-operatório (ayuno, inanición, periodo preoperatorio, cuidado preoperatorio, fasting, starvation, preoperative period, preoperative care). Na busca foram selecionados apenas estudos com adultos e idosos, sendo excluídas as gestantes, crianças e os adolescentes. Os dados foram analisados pelas pesquisadoras, segundo Gil $^{7}$ através das leituras exploratória, seletiva, analítica e interpretativa.

\section{RESULTADOS E DISCUSSÃO}

As buscas resultaram em um total de 189 artigos, sendo excluídos 149 através da leitura exploratória. A partir da leitura seletiva dos 40 artigos selecionados, foram excluídos 5 artigos por não se adequarem à questão da pesquisa do presente estudo. A leitura 
TABELA 1 - Consequências da abreviação do jejum pré-operatório e da resposta orgânica ao trauma.

\begin{tabular}{|c|c|c|}
\hline Autor/Ano & Delineamento - População e amostra & Principais achados \\
\hline $\begin{array}{l}\text { Kaška et } \\
\text { al. }^{20}(2010)\end{array}$ & $\begin{array}{l}\text { Estudo randomizado e controlado. Total de } 221 \text { pacientes } \\
\text { eletivos em cirurgia colorretal, divididos em três grupos. }\end{array}$ & $\begin{array}{l}\text { Jejum pré-operatório não confere benefício aos pacientes cirúrgicos. A } \\
\text { ingestão por via oral antes da cirurgia não aumenta o volume residual } \\
\text { gástrico e não foi associada com risco. }\end{array}$ \\
\hline $\begin{array}{l}\text { Oliveira et } \\
\text { al. }^{5}(2009)\end{array}$ & $\begin{array}{l}\text { Foram avaliados prospectivamente } 375 \text { pacientes submetidos } \\
\text { a diversas operações sobre o trato digestivo e parede } \\
\text { abdominal dentro de um novo protocolo. }\end{array}$ & $\begin{array}{l}\text { A adoção de medidas multidisciplinares perioperatórias do projeto ACERTO } \\
\text { não trouxeram nenhuma complicação relacionada ao jejum pré-operatório. }\end{array}$ \\
\hline $\begin{array}{l}\text { Melis et al. }{ }^{13} \\
\quad(2006)\end{array}$ & $\begin{array}{l}\text { Ensaio clínico, randomizado e controlado. Total de } 30 \\
\text { pacientes, divididos em } 3 \text { grupos de } 10 \text { pacientes cada. }\end{array}$ & $\begin{array}{l}\text { A ingestão pré-operatória de uma bebida rica em carboidratos pode } \\
\text { prevenir a imunodepressão e reduzir o risco de complicações infecciosas. }\end{array}$ \\
\hline $\begin{array}{l}\text { Yoshimasa et } \\
\text { al. }{ }^{24}(2011)\end{array}$ & $\begin{array}{l}\text { Estudo realizado com } 122 \text { pacientes de cirurgia eletiva, com } \\
\text { idade entre } 20-89 \text { anos, índice de massa corporal (IMC) } 18- \\
35 \mathrm{Kg} / \mathrm{m}^{2} \text {, divididos em dois grupos. }\end{array}$ & $\begin{array}{l}\text { A suplementação de bebida com } \mathrm{CHO} \text { no pré-operatório reduziu o } \\
\text { desconforto do paciente reduzindo o estresse antes da cirurgia. Um menor } \\
\text { tempo de jejum pode reduzir o desconforto associado a um procedimento } \\
\text { cirúrgico. }\end{array}$ \\
\hline $\begin{array}{l}\text { Aguilar- } \\
\text { Nascimento } \\
\text { et al. }{ }^{9}(2010)\end{array}$ & $\begin{array}{l}\text { Estudo de coorte histórica não aleatório realizado na } \\
\text { enfermaria de cirurgia de um hospital, com } 117 \text { pacientes com } \\
\text { idade igual ou superior a } 60 \text { anos, submetidos a operações } \\
\text { abdominais eletivas sob um protocolo convencional ou um } \\
\text { protocolo multimodal denominado ACERTO. }\end{array}$ & $\begin{array}{l}\text { A implantação do protocolo ACERTO foi acompanhada de queda do tempo } \\
\text { de jejum pré-operatório, do dia de realimentação pós-operatória e do } \\
\text { volume de fluídos intravenosos, da hospitalização e da infecção de sitio } \\
\text { cirúrgico em pacientes idosos submetidos a operações abdominais. }\end{array}$ \\
\hline $\begin{array}{l}\text { Svanfeldt et } \\
\text { al. }^{23}(2005)\end{array}$ & $\begin{array}{l}\text { Estudo randomizado cruzado, com } 6 \text { voluntários saudáveis } \\
\text { submetidos a quatro protocolos em uma ordem de } \\
\text { cruzamento aleatório. }\end{array}$ & $\begin{array}{l}\text { Uma bebida rica em carboidratos aumenta à ação da insulina 3h mais tarde, } \\
\text { por aproximadamente } 50 \% \text {. }\end{array}$ \\
\hline $\begin{array}{l}\text { Wang et al. }{ }^{11} \\
\qquad(2010)\end{array}$ & $\begin{array}{l}\text { Ensaio clínico randomizado, com } 52 \text { pacientes submetidos de } \\
\text { forma eletiva à ressecção de câncer colorretal. }\end{array}$ & $\begin{array}{l}\text { A ingestão de } \mathrm{CHO} \text { no pré-operatório melhora as sensações subjetivas de } \\
\text { fome e sede em comparação com o jejum enquanto atenua a resistência à } \\
\text { insulina. }\end{array}$ \\
\hline $\begin{array}{l}\text { Faria et al. }^{8} \\
\quad(2009)\end{array}$ & $\begin{array}{l}\text { Estudo clínico controlado e randomizado, com } 21 \text { candidatos } \\
\text { do sexo feminino (18-65 anos) para colecistectomia } \\
\text { laparoscópica. }\end{array}$ & $\begin{array}{l}\text { A abreviação do jejum no pré-operatório e a administração de uma bebida } \\
\text { contendo carboidratos diminui a resistência à insulina e melhora a resposta } \\
\text { ao trauma. }\end{array}$ \\
\hline $\begin{array}{l}\text { Dock- } \\
\text { Nascimento et } \\
\text { al. }{ }^{15}(2012)\end{array}$ & $\begin{array}{l}\text { Estudo clínico controlado randomizado, com } 48 \text { pacientes do } \\
\text { sexo feminino ( } 19-62 \text { anos) randomizadas para o grupo jejum } \\
\text { padrão (grupo controle) ou aos } 3 \text { grupos de diferentes bebidas } \\
\text { antes da vídeocolecistectomia. }\end{array}$ & $\begin{array}{l}\text { A abreviação do jejum pré-operatório com uma bebida de } \mathrm{CHO} \text { enriquecida } \\
\text { com glutamina parece melhorar a resistência à insulina e as defesas } \\
\text { antioxidantes além de diminuir a resposta inflamatória após a } \\
\text { vídeocolecistectomia. }\end{array}$ \\
\hline $\begin{array}{l}\text { Dock- } \\
\text { Nascimento et } \\
\text { al. }^{14}(2011)\end{array}$ & $\begin{array}{l}\text { Ensaio clínico controlado, duplo cego, randomizado, com } 56 \\
\text { mulheres (17-65 anos) submetidas à colecistectomia } \\
\text { laparoscópica eletiva. }\end{array}$ & $\begin{array}{l}\text { A abreviação do jejum pré-operatório para } 2 \mathrm{~h} \text { com } \mathrm{CHO} \text { e L-glutamina é } \\
\text { seguro e não aumenta o volume residual gástrico durante a indução da } \\
\text { anestesia. }\end{array}$ \\
\hline $\begin{array}{l}\text { Awad et al. } \\
\quad(2011)\end{array}$ & $\begin{array}{l}\text { Estudo randomizado, cruzado e duplo-cego, com } 12 \\
\text { voluntários saudáveis do sexo masculino de idade entre } 18 \text { a } \\
35 \text { anos, com um índice de massa corporal de } 20 \text { a } 25 \mathrm{~kg} / \mathrm{m}^{2} \text {. }\end{array}$ & $\begin{array}{l}\text { A ingestão de bebidas à base de carboidratos atenua a resistência à } \\
\text { insulina no pós-operatório e leva à diminuição de náuseas, vômitos, além } \\
\text { de manter a força muscular no pós-operatório e diminui o tempo de } \\
\text { internação hospitalar. A adição de glutamina e antioxidantes ao } \mathrm{CHO} \text { pode } \\
\text { beneficiar, pois melhoram a perfusão gastrointestinal e função imunológica. }\end{array}$ \\
\hline $\begin{array}{l}\text { Manchikanti et } \\
\text { al. }{ }^{19}(2011)\end{array}$ & $\begin{array}{l}\text { Estudo prospectivo, com } 3.179 \text { pacientes submetidos a } \\
\text { diferentes processos cirúrgicos. }\end{array}$ & $\begin{array}{l}\text { As reações pós-operatórias, como náuseas, vômitos e depressão } \\
\text { respiratória foram extremamente raras e a aspiração foi quase inexistente. }\end{array}$ \\
\hline $\begin{array}{l}\text { Hausel et al. } \\
\qquad(2005)\end{array}$ & $\begin{array}{l}\text { Ensaio clínico randomizado, com } 172 \text { pacientes submetidos à } \\
\text { colecistectomia laparoscópica eletiva. }\end{array}$ & $\begin{array}{l}\text { Carboidratos podem ter um efeito benéfico sobre náuseas e vômitos no } \\
\text { pós-operatório (12-24h) após a colecistectomia videolaparoscópica. }\end{array}$ \\
\hline $\begin{array}{l}\text { Scarlett et al. }{ }^{17} \\
\qquad(2002)\end{array}$ & Revisão sistemática. & $\begin{array}{l}\text { A aplicação das diretrizes e a ingestão de líquidos não resultou em aumento } \\
\text { da aspiração pulmonar, morbidade e mortalidade. Houve diminuição da } \\
\text { irritabilidade, ansiedade, sede e fome. }\end{array}$ \\
\hline $\begin{array}{l}\text { Gokhan et al. } \\
\qquad(2008)\end{array}$ & $\begin{array}{l}\text { Ensaio clínico controlado randomizado, com } 70 \text { pacientes } \\
\text { internados para colecistectomia ou tireoidectomia. }\end{array}$ & $\begin{array}{l}\text { A ingestão de } \mathrm{CHO} \text { no pré-operatório não parece alterar o volume ou o pH } \\
\text { do conteúdo gástrico, o que torna este procedimento seguro em termos de } \\
\text { risco de aspiração. }\end{array}$ \\
\hline $\begin{array}{l}\text { Järvelä et al. }{ }^{26} \\
\qquad(2008)\end{array}$ & $\begin{array}{l}\text { Ensaio clínico controlado randomizado, com } 101 \text { pacientes } \\
\text { agendados para CRM, randomizados em dois grupos: } \\
\text { controle (jejum na manhã do procedimento) e intervenção } \\
\text { (receberam bebida rica em } \mathrm{CHO} \text { ). }\end{array}$ & $\begin{array}{l}\text { É seguro permitir que pacientes de cirurgia cardíaca recebam uma bebida } \\
\text { clara com } \mathrm{CHO} \text { até } 2 \mathrm{~h} \text { antes da indução da anestesia, pois o esvaziamento } \\
\text { gástrico foi quase total e não houve aspiração. } \mathrm{A} \text { ingestão de bebida com } \\
\mathrm{CHO} \text { no pré-operatório por via oral não reduziu a resistência à insulina no } \\
\text { pós-operatório ou a ocorrência de náuseas ou vômitos. }\end{array}$ \\
\hline $\begin{array}{l}\text { Helminen et } \\
\text { al. }^{21}(2009)\end{array}$ & $\begin{array}{l}\text { Estudo prospectivo randomizado, com } 200 \text { pacientes } \\
\text { submetidos à cirurgia geral ou gastrointestinal, distribuídos } \\
\text { aleatoriamente em três grupos: infusão de glicose durante a } \\
\text { noite; bebida } \mathrm{CHO} \text { na manhã do procedimento ou jejum } \\
\text { durante a noite. }\end{array}$ & $\begin{array}{l}\text { A infusão intravenosa de glicose não diminui a sensação de sede e fome de } \\
\text { forma tão eficaz como uma bebida rica em carboidratos. }\end{array}$ \\
\hline $\begin{array}{l}\text { Henriksen et } \\
\text { al. }^{12}(2003)\end{array}$ & $\begin{array}{l}\text { Ensaio clínico controlado, randomizado, com } 48 \text { pacientes } \\
\text { submetidos a ressecções intestinais. }\end{array}$ & $\begin{array}{l}\text { Houve melhor resistência muscular no pós-operatório no grupo que recebeu } \\
\mathrm{CHO} \text { antes da cirurgia. Não houve diferenças no tempo de deambulação e } \\
\text { ingestão nutricional entre os grupos. }\end{array}$ \\
\hline $\begin{array}{l}\text { Bopp et al. }{ }^{27} \\
(2011)\end{array}$ & $\begin{array}{l}\text { Ensaio clínico randomizado prospectivo, com } 123 \text { pacientes } \\
\text { submetidos à cirurgia oftalmológica e } 109 \text { pacientes foram } \\
\text { aleatoriamente atribuídos a um dos dois regimes de jejum pré- } \\
\text { operatório. }\end{array}$ & $\begin{array}{l}\text { A padronização de ingestão limitada de líquidos por via oral no pré- } \\
\text { operatório aumenta o conforto e a satisfação do paciente com os cuidados } \\
\text { da anestesia em cirurgias oftalmológicas. }\end{array}$ \\
\hline $\begin{array}{l}\text { Noblett et al. } \\
\quad(2006)\end{array}$ & $\begin{array}{l}\text { Ensaio clínico randomizado, com } 36 \text { pacientes submetidos à } \\
\text { ressecção do cólon em um dos três grupos: grupo } 1 \text { (jejum), } \\
\text { grupo } 2 \text { (água por via oral pré-operatória), grupo } 3 \text { (bebida } \\
\text { rica em } \mathrm{CHO} \text { ). }\end{array}$ & $\begin{array}{l}\text { Administração pré-operatória oral de carboidratos leva a uma redução } \\
\text { significativa na internação pós-operatória e um retorno mais cedo da função } \\
\text { intestinal quando comparado com o jejum ou água. }\end{array}$ \\
\hline
\end{tabular}


analítica foi realizada em 35 artigos relacionando o seu conteúdo com as questões de pesquisa e resultando em 20 estudos nos quais foi realizada a leitura interpretativa. Na maioria foram verificados os efeitos da abreviação do jejum através da ingestão de bebidas ricas em $\mathrm{CHO}$, acrescidas ou não de imunonutrientes.

Os resultados foram organizados em uma categoria, denominada "Consequências da abreviação do jejum pré-operatório e da resposta orgânica ao trauma", e seis temas relacionados, como: 1.1 Resistência à insulina; 1.2 Bebidas com carboidratos; 1.3 Bebidas com carboidratos e proteínas; 1.4 Náuseas, vômitos e aspiração; 1.5 Esvaziamento gástrico e pH gástrico; 1.6 Conforto do paciente. Na tabela 1 encontra-se o resumo dos estudos selecionados.

\section{Consequências da abreviação do jejum pré-operatório e da resposta orgânica ao trauma}

Deve-se considerar que a resposta endócrina e metabólica ao trauma cirúrgico pode ser aumentada pelo jejum pré-operatório prolongado. Após algumas horas de jejum, os índices de insulina diminuem e os de glucagon se elevam. Os níveis séricos do hormônio do crescimento também se elevam quando há hipoglicemia ou diminuição na circulação de ácidos graxos livres ${ }^{8}$.

Nas últimas décadas, resultados animadores com a aplicação de programas multimodais objetivando a otimização da recuperação pós-operatória têm feito com que velhos paradigmas sejam questionados dando lugar a práticas mais modernas, sustentadas em evidências. Pacientes a serem operados pela manhã (depois das seis horas) são orientados a guardar jejum para alimentos sólidos a partir das 22 horas da véspera, o que corresponde a um período de jejum não inferior a oito horas. Pequenas quantidades de água acompanhando a medicação pré-anestésica ou outros medicamentos administrados por via oral, não oferecem inconveniente e são permitidos. Embora o jejum de duas horas para líquidos claros seja recomendado, na prática poucos serviços o adotam. Conforme estudo de Oliveira et $\mathrm{al}^{5}$, em que o objetivo foi avaliar o surgimento de possíveis complicações anestésicas relacionadas à abreviação do jejum pré-operatório para duas horas com uma solução contendo dextrinomaltose a $12,5 \%$ dentro do projeto ACERTO (Aceleração da Recuperação Total Pós-operatória), conclui-se que foi possível a redução do tempo de jejum pré-operatório com a suplementação nutricional para o paciente sem diminuir a segurança para o procedimento anestésico ${ }^{5}$.

Estudos têm mostrado que várias rotinas de cuidados perioperatórios são inúteis e em alguns casos, prejudiciais. Além disso, no período perioperatório o suporte nutricional é frequentemente negligenciado. Protocolos na Europa (ERAS - Enhanced Recovery After Surgery) e no Brasil (ACERTO) têm demonstrado que a adoção de estratégias multimodais de cuidado perioperatório estão associadas com menor tempo de hospitalização pós-operatório e diminuição de morbidade infecciosa. Conforme estudo publicado por Aguilar-Nascimento et al. ${ }^{9}$ os pacientes idosos são mais propensos a complicações pós-operatórias, os quais geralmente são candidatos a grandes operações, e são mais susceptíveis a variações na homeostase. Além disso, eles têm frequentemente comorbidades, tais como diabetes, hipertensão arterial, doença pulmonar crônica e complicações cardiovasculares. O estudo teve como objetivo comparar a evolução clínica antes e depois da aplicação de um protocolo multimodal perioperatório (ACERTO) em uma população de idosos que foram submetidos a operações abdominais. Os resultados mostraram que a implantação de rotinas multidisciplinares do protocolo ACERTO diminuiu a hospitalização e a infecção de sitio cirúrgico em pacientes idosos submetidos a operações abdominais. Esses benefícios foram mostrados em vários parâmetros pós-operatórios, como redução no tempo de jejum pré-operatório, queda do dia de realimentação pós-operatória, diminuição do volume de fluidos intravenosos, menor tempo de internação e morbidade. A implementação de estratégias multimodais podem proporcionar benefícios claros para pacientes idosos submetidos a operações abdominais, principalmente no trato digestivo ${ }^{9}$.

\subsection{Resistência à insulina}

A resistência à insulina no pós-operatório é um fenômeno transitório, com duração de até três semanas após a realização de operações abdominais eletivas e 
não complicadas. Ela aumenta conforme aumenta o porte da operação, mesmo ocorrendo em cirurgias de pequeno e moderado porte, como herniorrafias e colecistectomias videolaparoscópicas (CVL). Nesta situação, a captação de glicose através das células diminui pela incapacidade do transportador GLUT-4 realizar a ação. Como consequência, a produção de glicogênio diminui. Ao mesmo tempo, há aumento da produção, de forma endógena, de glicose pela neoglicogênese, de maneira que a glicemia sanguínea encontra-se elevada. $O$ efeito adverso da hiperglicemia tem sido elucidado recentemente, e uma relação causal entre resistência à insulina no pós-operatório e as complicações após a cirurgia tem sido sugeridas, o que promove grave risco para morbidade e mortalidade.

O grau de resistência à insulina é considerado como sendo um fator independente e determinante no tempo de internação hospitalar pós-operatória. Sabe-se que quanto maior a resistência à insulina, maior é o tempo de internação hospitalar do paciente ${ }^{10}$. Estudo realizado por Wang et al. ${ }^{11}$ demonstrou que os pacientes que receberam uma bebida com $\mathrm{CHO}$ antes da ressecção eletiva do câncer colorretal reduziram a resistência à insulina, além de apresentar menos sede e fome no préoperatório do que os pacientes que jejuaram durante a noite. Estes resultados sugerem que uma bebida de carboidratos antes da cirurgia colorretal tem efeito clinicamente benéfico no que diz respeito ao seu efeito sobre a sensibilidade à insulina ${ }^{11}$.

Em outro estudo, Faria et al. ${ }^{8}$ investigou a influência sobre a resistência à insulina e resposta metabólica ao trauma após administração de uma bebida rica em $\mathrm{CHO}$ dado $2 \mathrm{~h}$ antes da CVL. No geral, os resultados deste estudo mostram que um período reduzido do tempo de jejum pré-operatório com a entrega de carboidratos não só diminui a resistência à insulina no pós-operatório, mas também reduz vários parâmetros bioquímicos relacionados à resposta metabólica ao trauma. $\mathrm{O}$ jejum convencional de $8 \mathrm{~h}$ foi associado ao aumento dos níveis de glicose e insulina. Os novos dados fornecidos por este estudo mostram claramente que a resistência à insulina é reduzida sob estas circunstâncias. Os resultados também apoiam a mudança de velhos hábitos compartilhados por cirurgiões e anestesiologistas. Os resultados globais permitem-nos concluir que um reduzido período de jejum préoperatório com bebida de carboidratos diminui a resistência à insulina e a resposta metabólica ao trauma após $\mathrm{CVL}^{8}$.

\subsection{Bebidas com carboidratos}

Rígidos períodos de jejum, iguais ou superiores a 8 horas, passaram a ser substituídos por regimes mais liberais. Atualmente, a American Society of Anesthesiologists (ASA) recomenda diretrizes mais flexíveis em relação ao jejum, liberando o uso de líquidos claros (água, chá, café e sucos sem resíduos), até 2 horas antes da operação. A possibilidade de abreviar o jejum pré-operatório motivou a busca por soluções de substratos energéticos de rápida absorção e que não interferem no esvaziamento gástrico ${ }^{10}$.

A partir de novas publicações, trabalhos surgiram evidenciando que o uso de uma solução de líquido enriquecida com $\mathrm{CHO}$ determina maior satisfação do paciente, menor irritabilidade do paciente, aumento do $\mathrm{pH}$ gástrico e principalmente, reduz a resposta catabólica ao estresse cirúrgico, com consequente melhora da recuperação pós-operatória ${ }^{10}$. Através da possibilidade de uso de líquidos claros até 2 horas antes da operação, a associação com $\mathrm{CHO}$ mostra-se benéfica com a finalidade de reverter a resistência à insulina relacionada ao trauma cirúrgico ${ }^{8}$.

Atualmente, na Europa, usa-se uma dose de $400 \mathrm{~mL}$ de bebida enriquecida com maltodextrina $(50 \mathrm{~g})$, de modo bastante seguro. Alguns estudos randomizados mostraram que o uso de $800 \mathrm{~mL}$ dessa bebida na noite que antecede a operação e $400 \mathrm{~mL}$ cerca de 2 horas antes da operação reduzem em $50 \%$ a resistência à insulina em cirurgias colorretais de grande porte. Faria et al. $^{8}$ mostraram que essa redução é possível com somente $200 \mathrm{~mL}$ de maltodextrina a $12,5 \% 2 \mathrm{~h}$ antes da realização da $\mathrm{CVL}^{8}$. Além desse efeito, há estudos demonstrando os benefícios dessa conduta na perda de massa e força musculares no pós-operatório. Henriksen et al. $^{12}$ evidenciaram que houve melhora na resistência muscular no pós-operatório no grupo que recebeu $\mathrm{CHO}$ antes da cirurgia ${ }^{12}$. Outros benefícios do uso de soluções no pré-operatório são a redução da ansiedade 
e da fome nos pacientes, como demonstrado no estudo de Melis et al. ${ }^{13}$ em que investigaram se a ingestão de bebidas ricas em $\mathrm{CHO}$ no pré-operatório pode influenciar no sistema imunológico. O estudo sugeriu que a ingestão pré-operatória de uma bebida rica em $\mathrm{CHO}$ pode prevenir a imunodepressão e, portanto, reduzir o risco de complicações infecciosas, bem como proporcionar maior bem estar nos pacientes, comparados aquele grupo que não recebeu a solução de $\mathrm{CHO}^{13}$.

\subsection{Bebidas com carboidratos e proteínas}

A adição de proteínas ou aminoácidos diminui ainda mais a resistência à insulina, a perda de massa magra $e$ consequentemente a resposta orgânica ao trauma. Estudos randomizados, associando glutamina ou proteína hidrolisada à bebida contendo $\mathrm{CHO}$ observaram segurança do esvaziamento gástrico.

A utilização de agentes de condicionamento, tais como a glutamina pode trazer benefício para pacientes submetidos à cirurgia. A Glutamina é um aminoácido condicional, que melhora a perfusão gastrointestinal e a função imunitária. A bebida contendo glutamina além de $\mathrm{CHO}$ pode fornecer benefícios adicionais para os pacientes cirúrgicos. Dock - Nascimento et al. ${ }^{14}$ testaram uma bebida com maltodextrina e glutamina e observaram que o volume residual gástrico (VRG) era semelhante em pacientes que ficaram em jejum ou ingeriram apenas maltodextrina ${ }^{14}$. Em outro estudo randomizado, Dock-Nascimento et al. ${ }^{15}$ avaliou se houve melhora na resposta biológica após a CVL. A abreviação do jejum pré-operatório com uma bebida de $\mathrm{CHO}$ enriquecida com glutamina parece melhorar a resistência à insulina e as defesas antioxidantes, além de diminuir a resposta inflamatória após a operação.

Os resultados sugerem que a diminuição do tempo de jejum pré-operatório pode induzir um ambiente favorável para o pós-operatório, e a suplementação de glutamina associada ao $\mathrm{CHO}$ pode melhorar o resultado ${ }^{15}$.

\subsection{Náuseas, vômitos e risco de aspiração}

A incidência de náuseas e vômitos no pós-operatório é menor em pacientes que ingeriram solução de $\mathrm{CHO}$ $2 \mathrm{~h}$ antes da operação. Hausel et al. ${ }^{16}$ demonstraram menor índice de náuseas e vômitos com 12 a 24 horas de pós-operatório em pacientes que usaram a solução de $\mathrm{CHO}$ antes da $\mathrm{CVL}^{16}$. Dentre os pacientes de risco para aspiração pulmonar do conteúdo gástrico, aqueles com refluxo gastroesofágico merecem cuidados adicionais. Por essa razão esses pacientes foram excluídos do protocolo de abreviação do jejum préoperatório ${ }^{5}$.

Estudos experimentais e revisões têm mostrado consistentemente a segurança da ingestão de líquidos claros até $2 \mathrm{~h}$ antes da indução anestésica. Scarlett et al. ${ }^{17}$ mostraram que a aplicação das diretrizes para abreviação do jejum pré-operatório e a ingestão de líquidos não resultou em aumento dos casos de aspiração pulmonar, morbidade e mortalidade na população estudada. Além disso, houve diminuição da irritabilidade, ansiedade, sede e fome no período préoperatório $^{17}$.

Os principais fatores associados à ocorrência de aspiração brônquica na indução anestésica são a operação de urgência, volume gástrico alto, acidez gástrica ( $\mathrm{pH}$ baixo), pressão intra-abdominal, refluxo gastresofágico, doença esofágica, trauma craniano com comprometimento da consciência e idade baixa ou avançada $^{17}$.

\subsection{Esvaziamento gástrico e pH gástrico}

Estudos demonstraram que o jejum prolongado resulta num maior volume residual gástrico, com $\mathrm{pH}$ ainda mais ácido, enquanto a ingestão de líquidos claros até duas horas antes do procedimento, torna o volume residual menor e o pH maior. Somam-se a este argumento constatações de que o jejum pré-operatório prolongado, além de bastante desconfortável e desnecessário, pode ser prejudicial ao potencializar ou perpetuar a resposta orgânica ao trauma ${ }^{5}$.

Em estudo randomizado e controlado, Gokhan et al. ${ }^{18}$ concluíram que o uso de liquido oral, enriquecido com $\mathrm{CHO}(12,5 \mathrm{~g}$ de carboidrato para cada $100 \mathrm{~mL}, 12 \%$ monossacarídeo, $12 \%$ dissacarídeo e $76 \%$ polissacarídeo, $285 \mathrm{mOsm} / \mathrm{Kg}$ ), na dose de $800 \mathrm{~mL}$ na noite da véspera da operação, e de $400 \mathrm{~mL} 2$ horas antes da indução anestésica, não altera $\circ \mathrm{pH}$ gástrico nem o seu conteúdo, sugerindo segurança em termos 
de risco de broncoaspiração ${ }^{18}$.

\subsection{Conforto do paciente}

Algumas variáveis psicológicas como ansiedade, depressão e dor têm sido associadas como preditivo de maus resultados cirúrgicos. A ansiedade é uma reação emocional definida como tensão, apreensão, preocupação e nervosismo, causada algumas vezes por uma ameaça intangível. Pacientes ansiosos podem não cooperar durante a realização das técnicas intervencionistas e não só podem não responder bem como podem complicar os procedimentos ${ }^{19}$.

Kaska et al. ${ }^{20}$ comparou o jejum com a administração de $\mathrm{CHO}$, minerais e água via oral nas cirurgias colorretais. A administração de $\mathrm{CHO}$ no pré-operatório levou a diminuição no tempo de internação hospitalar, melhora da função intestinal quando comparados ao grupo que realizou jejum prolongado. As queixas relacionadas às condições psicossomáticas de sede, fome, ansiedade e dor foram significativamente menores no grupo que recebeu $\mathrm{CHO}$ no período pré-operatório, tornando a recomendação segura e melhorando o bem estar subjetivo do paciente antes da operação ${ }^{20}$.

As evidências encontradas na revisão de literatura em relação à abreviação do jejum pré-operatório têm feito com que velhos paradigmas sejam questionados dando lugar a práticas mais modernas. Várias Sociedades de Anestesiologia recomendam $2 \mathrm{~h}$ de jejum pré-operatório para líquidos claros e $6 \mathrm{~h}$ de jejum para sólidos para a maioria dos pacientes submetidos à cirurgia eletiva. Soluções que contenham carboidratos e outros nutrientes são promissoras quanto à melhora na resposta endócrino-metabólica ao trauma. A satisfação do paciente também deve ser considerada, e é maior quando se empregam períodos mais curtos de jejum no pré-operatório.

\section{REFERÊNCIAS}

1. Ferreira, Aurélio Buarque de Holanda. Dicionário Aurélio Básico da Língua Portuguesa. Curitiba: Positivo, 2008, p. 302

2. Lameu E. Inanição. In: Lameu E. Clínica nutricional. Rio de Janeiro: Revinter; 2005. p.153-60

3. Bozzetti, F, Forbes A. The ESPEN clinical practice guidelines on Parenteral Nutrition: Present status and perspectives for future research. Clin Nutr. 2009 Aug;28:359-64.
4. Nygren J. The metabolic effects of fasting and surgery. Best Pract Res Clin Anaesthesiol. 2006 Sep; 20:429-438.

5. Oliveira KG, Balsan M, Oliveira Sde S, Aguilar-Nascimento JE. A abreviação do jejum pré-operatório para duas horas com carboidratos aumenta o risco anestésico? Rev Bras Anestesiol. 2009 Sep-Oct;59(5):577-84.

6. Aguilar-Nascimento JE, Bicudo-Salomão A, Caporossi C Silva RM, Cardoso EA, Santos TP. Acerto pós-operatório: avaliação dos resultados da implantação de um protocolo multidisciplinar de cuidados perioperatórios em cirurgia geral. Rev Col Bras Cir. 2006 Jun;33:181-8.

7. Gil AC. Como delinear uma pesquisa bibliográfica In: Gil AC. Como elaborar projetos de pesquisa. São Paulo: Atlas; 2002. p. 59-85.

8. Faria MSM, Aguilar-Nascimento JE, Pimenta OS, Alvarenga LC Jr, Dock-Nascimento DB, Slhessarenko N. Preoperative fasting of 2 hours minimizes insulin resistance and organic response to trauma after videocholecystectomy: a randomized, controlled, clinical trial. World J Surg. 2009 Jun;33(6):1158-64.

9. Aguilar-Nascimento JE, Salomão AB, Caporossi C, Diniz BN. Clinical benefits after the implementation of a multimodal perioperative protocol in elderly patients. Arq Gastroenterol. 2010 Apr-Jun;47(2):178-83.

10. Salomão AB, Aguilar-Nascimento JE. Jejum préoperatório. In: Aguilar-Nascimento JE, Caporossi C, Salomão AB. Acerto: acelerando a recuperação total pósoperatória. 2aed. Rio de Janeiro: Editora Rubio; 2011. p.73-87.

11. Wang ZG, Wang Q, Wang WJ, Qin HL. Randomized clinical trial to compare the effects of preoperative oral carbohydrate versus placebo on insulin resistance after colorectal surgery. Br J Surg. 2010 Mar;97(3):317-27.

12. Henriksen MG, Hessov I, Dela F, Hansen HV, Haraldsted $\mathrm{V}$, Rodt SÅ. Effects of preoperative oral carbohydrates and peptides on postoperative endocrine response, mobilization, nutrition and muscle function in abdominal surgery. Acta Anaesthesiologica Scandinavica. 2003 Feb;47(2):191-199.

13. Melis GC, Van Leeuwen PA, Von Blomberg-van der Flier BM, Goedhart-Hiddinga AC, Uitdehaag BM, Strack van Schijndel RJ, Wuisman PI, van Bokhorst-de van der Schueren MA. A carbohydrate-rich beverage prior to surgery prevents surgery-induced immunodepression: a randomized, controlled, clinical trial. JPEN J Parenter Enteral Nutr. 2006 Jan-Feb;30(1):21-6.

14. Dock-Nascimento DB, Aguilar-Nascimento JE, Caporossi C, Sepulveda Magalhães Faria M, Bragagnolo $R$, Caporossi FS, Linetzky Waitzberg D. Safety of oral glutamine in the abbreviation of preoperative fasting: a double-blind, controlled, randomized clinical trial. Nutr Hosp. 2011 Jan-Feb;26(1):86-90.

15. Dock-Nascimento DB, de Aguilar-Nascimento JE, Magalhaes Faria MS, Caporossi C, Slhessarenko N, Waitzberg DL. Evaluation of the effects of a preoperative 2-hour fast with maltodextrine and glutamine on insulin resistance, acute-phase response, nitrogen balance, and serum glutathione after laparoscopic cholecystectomy: a controlled randomized trial. JPEN J Parenter Enteral Nutr. 2012 Jan;36(1):43-52.

16. Hausel J., Nygren J., Thorell A., Lagerkranser M. and Ljungqvist $O$. Randomized clinical trial of the effects of oral preoperative carbohydrates on postoperative nausea and vomiting after laparoscopic cholecystectomy. British Journal of Surgery. 2005 Apr;92(4):415-421.

17. Scarlett M, Crawford-Sykes A, Nelson M. Preoperative starvation and pulmonary aspiration: New perspectives and guidelines. West Indian Medical Journal. 2002 Dec;51(4):241-245.

18. Gokhan Y, Mehmet FC, Erkan O, Birgul D, Taher O, 
Ahrmet C, Turgut T. Effects of preoperative carbohydrate loading on glucose metabolism and gastric contents in patients undergoing moderate surgery: a randomized, controlled trial. Nutrition. 2008 Mar;24(3):212-6.

19. Manchikanti L, Malla Y, Wargo BW, Fellows B Preoperative fasting before interventional techniques: is it necessary or evidence-based? Pain Physician. 2011 SepOct;14(5):459-67.

20. Kaska M, Grosmanová T, Havel E, Hyspler R, Petrová Z, Brtko M, Bares P, Bares D, Schusterová B, Pyszková L, Tosnerová V, Sluka M. The impact and safety of preoperative oral or intravenous carbohydrate administration versus fasting in colorectal surgery--a randomized controlled trial. Wien Klin Wochenschr. 2010 Jan;122(1-2):23-30.

21. Helminen $\mathrm{H}$, Viitanen $\mathrm{H}$, Sajanti J. Effect of preoperative intravenous carbohydrate loading on preoperative discomfort in elective surgery patients. Eur J Anaesthesiol. 2009 Feb;26(2):123-7.

22. Noblett SE, Watson DS, Huong H, Davison B, Hainsworth PJ, Horgan AF. Pre-operative oral carbohydrate loading in colorectal surgery: A randomized controlled trial. Colorectal Disease. 2006 Sep;8(7):563-569.

23. Svanfeldt $M$, Thorell A, Hausel J, Soop M, Nygren J, Ljungqvist $O$. Effect of "preoperative" oral carbohydrate treatment on insulin action - A randomised cross-over unblinded study in healthy subjects. Clinical Nutrition. 2005 Oct;24(5):815-821.
24. Oyama $Y$, Iwasaka H, Shiihara K, Hagiwara S, Kubo N, Fujitomi $Y$, Noguchi T. Effects of preoperative oral carbohydrates and trace elements on perioperative nutritional status in elective surgery patients. Middle East $J$ Anesthesiol. 2011 Oct;21(3):375-83.

25. Awad S, Fearon KCH, Macdonald IA, Lobo DN. A randomized cross-over study of the metabolic and hormonal responses following two preoperative conditioning drinks. Nutrition. 2011 Sep;27(9):938-42.

26. Järvelä $K$, Maaranen $P$, Sisto $T$. Pre-operative oral carbohydrate treatment before coronary artery bypass surgery. Acta Anaesthesiol Scand. 2008 Jul;52(6):793-7. Epub 2008 May 12.

27. Bopp C, Hofer S, Klein A, Weigand MA, Martin E, Gust R. A liberal preoperative fasting regimen improves patient comfort and satisfaction with anesthesia care in day-stay minor surgery. Minerva Anestesiol. 2011 Jul;77(7):680-6.

\section{Endereço para correspondência:}

Patrícia da Fonseca Flores

Avenida Coronel Aparício Borges $n^{\circ}$ 390, apto. 401

Porto Alegre/RS - CEP 90680-570

Telefone: +55 5192794724

E-mail: patriciaflores_nutri@yahoo.com.br 